\title{
Osseointegration of Titanium Prostheses on the Stapes Footplate
}

\author{
Marcus Neudert $^{1}$, Thomas Beleites ${ }^{1}$, Michael Ney $^{2}$, Anne Kluge ${ }^{1}$, Nikoloz Lasurashvili ${ }^{1}$, \\ Matthias Bornitz $^{1}$, Dieter Scharnweber ${ }^{3}$, and Thomas Zahnert ${ }^{1}$ \\ ${ }^{1}$ Department of Otolaryngology, Head and Neck Surgery, Technische Universität Dresden, Fetscher Strasse 74, 01307 Dresden, \\ Germany \\ ${ }^{2}$ Institute of Pathology, Technische Universität Dresden, Fetscher Str. 74, 01307 Dresden, Germany \\ ${ }^{3}$ Max Bergmann Center of Biomaterials, Technische Universität Dresden, Budapester Str. 27, 01062 Dresden, Germany
}

Received: 3 March 2009; Accepted: 1 December 2009; Online publication: 12 January 2010

\begin{abstract}
The success of middle ear reconstructive surgery depends on stable coupling between the prosthesis and residual ossicles. To establish a stable fixed point on the stapes footplate for subsequent prosthesis reconstruction, a titanium footplate anchor was coated with osteoinductive substances to induce a controlled osseointegration on the footplate. Various studies have shown that collagen-based matrices with and without bone growth and differentiation factors can induce and enhance bone formation and consequently increase implant stability. The ears of 23 oneyear-old Merino sheep $(n=46)$ were divided into five groups and implanted with a specially designed footplate anchor. The surface of each implant was modified by applying a collagenous matrix (collagen I or II) either with immobilized bone morphogenic protein (BMP-4) or transforming growth factor- $\beta$, respectively, to stimulate osteoblastic activation and differentiation on the stapes footplate with subsequent osseointegration. Polychrome labeling was used to assess new bone formation and remodeling during the study. After study termination on day 84, synchrotron radiation-based computed microtomography and histomorphometry were used to identify bone implant contact. Eight implants showed radiographical and/or histological
\end{abstract}

This work was presented in part at the 78th Annual Meeting of the Deutsche Gesellschaft für HNO-Heilkunde Kopf- und Hals-Chirurgie, 2007, Munich, Germany.

Correspondence to: Marcus Neudert · Department of Otolaryngology, Head and Neck Surgery · Technische Universität Dresden · Fetscher Strasse 74, 01307 Dresden, Germany. Telephone: +49-351-4584420; fax: +49-351-4582264; email: marcus.neudert@uniklinikum-dresden.de evidence of integration by newly formed bone. An osseointegration could histologically be proven in two of these eight specimens, and additional ectopic bone formations were seen in another 21 specimens. In all animals, bone turnover on the footplate was proven by polychrome labeling. This study proves the general ability to induce a controlled osseointegration of titanium implants biologically activated with artificial extracellular matrices on their surfaces on the stapes footplate in a mammalian organism.

Keywords: tympanoplasty, reconstruction, animal study, growth factors, implant coating

Abbreviations: TORP - Total ossicular replacement prosthesis; Coll-Collagen; CS - Condroitin sulfate; DC-Decorin; BMP - Bone morphogenic protein; TGF- $\beta$ - Transforming growth factor- $\beta$; ABR Auditory brainstem response; FSL-Fluorochrome sequential labeling; SR $\mu$ CT - Synchrotron radiationbased computed microtomography

\section{INTRODUCTION}

Dislocation of prostheses and/or interposition of connective tissue can impede a direct prosthesis bone contact and are important reasons for poor postoperative sound transmission after ossiculoplasty (Hüttenbrink 1994; Katzke et al. 1982; Smyth 1983). Therefore, stable coupling between prostheses and the remaining ossicular rudiments is a precondition for satisfactory hearing results (Eiber et al. 2000). In 
the case of a destroyed stapes superstructure with only an intact footplate remaining in the oval niche, the interposition of a total ossicular replacement prosthesis (TORP) represents the current clinical practice. Sound transmission from the tympanic membrane to the inner ear is thereby enabled by a collumella prosthesis whose position on the stapes footplate is secured by pieces of connective tissue or cartilage (Fisch and May 1994; Jahnke 1987; Plester et al. 1989). However, an apparently reliably positioned TORP imminently runs the risk of dislocation at the end of surgery. Changes in atmospheric pressure, middle ear effusion, or scar tissue development can destroy the prosthesis fit on the footplate. Additionally, any interposition of elastic connective tissue in the prosthesis footplate interface decreases an effective sound transmission. For both of these reasons, an osseointegration of the prosthesis material on the footplate would be preferable.

Osseointegration has been used to define a direct structural and functional connection between ordered living bone and the surface of an implant (Branemark et al. 1977). Even though success rates of up to $95 \%$ for certain implants have been reported, in reconstructive middle ear surgery, the moving implant bed causes poor preconditions for a successful bony integration. Since primary implant stability is fundamental for developing implant osseointegration, a compound prosthesis concept is necessary to maintain implant stability in the first phases of integration. A two-step approach could secure primary implant stability by establishing a firm and stable titanium anchor on the footplate followed by surgically mounting the proper prosthesis for sound transmission.

However, the stapes footplate represents an unfavorable environment for successful osseointegration. The thin bony footplate layer holds only a small number of osteocytes, cells that are crucial for osseointegration. Additionally, in contrast to any other site of implant osseointegration, an iatrogenic bone defect for new bone formation as the starting stimulus is missing.

Like in other cases of a reduced implant take rate, e.g., in bone of poor tissue quality, metabolic bone disease or after radiotherapy, it is of considerable interest to enhance the quality and rate of new bone formation around implants. This can be achieved by modification of the implant surface, which in turn stimulates the active process of osseointegration. Types I and II collagen (coll), both major components of the extracellular matrix (ECM), are known for their binding properties for osteoblastic cells (Becker et al. 2002). Collagen-coated implants can additionally be modified by association with non-collagenous ECM components (Douglas et al. 2007a, b). The glycosaminoglycan chondroitin sulfate (CS) and the proteoglycan decorin (DC) enable such complex coll/ECM matrices to store and stabilize growth factors (Rosso et al. 2004) and thereby enhance the induction of osteogenic cell proliferation, differentiation, and growth.

Coll-I/CS- and coll-II/DC-coated implants can further be augmented by recombinant growth factors. The influence of bone morphogenetic proteins (BMP) and tissue growth factors (rhTGF- $\beta$ ) on induction of osteoblastic proliferation, differentiation, and bone formation in general and on peri-implant bone formation was shown in different studies (Bessho et al. 1999; Wong et al. 1995). These implant surface modifications were therefore used to induce and improve the bone integration of titanium implants on the stapes footplate by both the pre-integration of osteoinductive recombinant growth factors (rhBMP-4, rhTGF- $\beta$ ) and the possible interaction of ECM components with endogenous growth factors.

\section{MATERIAL AND METHODS}

\section{Animals}

Twenty-four 12-month-old female Merino sheep were obtained from and kept under standard conditions at a local breeder (Theinert, Canitz, Germany). All animals had free access to water and were fed ad libitum with a commercial standard chow containing $1.1 \mathrm{~g}$ calcium and $0.8 \mathrm{~g}$ phosphorus per $100 \mathrm{~g}$ dry weight (Schkade Landhandel GmbH, Belgern, Germany). For all experimental procedures (operation, brainstem-evoked response audiometry (BERA), and killing), except the polychrome labeling, animals were transported to the local center for animal experiments (MTZ, Dresden, Germany).

After adaptation to laboratory conditions for 2 days, the animals entered the study. Twelve hours prior to the operation, they were kept in double cages for fasting. At study termination, animals were killed at the end of an auditory brainstem response (ABR) measurement while under general anesthesia. Embutramide $(6 \mathrm{mg} / 50 \mathrm{~kg}$ body weight) was administered as a single injection in combination with tetracainhydrochloride and mebezoniumiodide $\left(\mathrm{T} 61^{\circledR}\right.$, Intervet Co, Unterschleißheim, Germany). All experiments were approved by the governmental Animals Ethic Committee (Regierungspräs, Dresden, Germany).

\section{Implants/footplate anchor}

A titanium implant (titanium medical grade II), hereafter called footplate anchor, was designed as an oval stamp with three bumps at the undersurface to improve the contact area on the individually varying footplate surface. These bumps were intended to act as starting zones for the tissue integration. The size of the oval base area was $0.55 \times 0.35 \mathrm{~mm}$, with a total height of $0.54 \mathrm{~mm}$. Including the three bumps 
(diameter $=0.13 \mathrm{~mm}$; area $=0.023 \mathrm{~mm}^{2}$ each $)$, the whole undersurface of the implant, which was subsequently coated as mentioned below, accounts for $0.425 \mathrm{~mm}^{2}$. The strut of the footplate anchor, to which the main prosthesis will be coupled, is simplified as a bar for handling during coating and operation. The implant mass was calculated for $185 \mu \mathrm{g}$. Geometrical data of the sheep's stapes and its footplate were obtained from micro-CT scans of an extracted stapes specimen (Müller et al. 2007). Titanium implants were manufactured by Fraunhofer Institute for Machine Tools and Forming Technology (Chemnitz, Germany). Footplate anchor geometry and its intended use in the human middle ear are shown in Figure 1.

\section{Coating of footplate anchor}

The following five surface conditions were used in the following experimental groups:

Group $1(n=8)$ : titanium (Ti), no coating

Group $2(n=9)$ : Ti+collagen I+chondroitine sulfate (coll-I/CS)

Group $3(n=10)$ : Ti+collagen I+chondroitine sulfate+rhBMP-4 (coll-I/CS/BMP-4)

Group 4 ( $n=10)$ : Ti+collagen II+decorin (coll-II/DC) Group $5(n=9)$ : Ti+collagen II+decorin+rhTGF- $\beta 1$ (coll-II/DC/TGF)

For preparation of coatings, collagen was dissolved at $5 \mathrm{mg} / \mathrm{ml}$ in $10 \mathrm{mM}$ acetic acid overnight at $4^{\circ} \mathrm{C}$. The collagen solution was then mixed on ice with equal volumes of twofold concentrated fibrillogenesis buffer $(60 \mathrm{mM}$ sodium phosphate, $270 \mathrm{mM} \mathrm{NaCl}, \mathrm{pH}$ 7.0). CS and decorin were added with $30 \mu \mathrm{g} / \mathrm{mg}$ collagen. Fibrillogenesis was allowed to take place overnight at $37^{\circ} \mathrm{C}$. The resulting gel was homogenized. Fibrils were collected by centrifugation at $5,000 \times g$ for $15 \mathrm{~min}$, washed with fibrillogenesis buffer diluted to working concentration, and centrifuged again. The pellet was resuspended in the same buffer to a concentration of about $5 \mathrm{mg} / \mathrm{ml}$ collagen. The implants were incubated in the suspension at $25^{\circ} \mathrm{C}$ for $5 \mathrm{~min}$ and air-dried. This process was repeated two times. The footplate anchor was kept in position by specially designed fixtures with the footplate anchor undersurface upside down. The footplate anchor strut was completely embedded in a silicone fitting to provide a stable fixation during coating. However, this only allowed the undersurface to be coated. The implants were then washed with distilled water and sterilized with ethylene oxide at $42^{\circ} \mathrm{C}$ for $12 \mathrm{~h}$ (Wolf et al. 1999). Growth factors were allowed to adsorb to the surfaces over night at $4^{\circ} \mathrm{C}$ with $100 \mathrm{ng} / \mathrm{ml} \mathrm{rhTGF}$ $\beta 1$ and $2 \mu \mathrm{g} / \mathrm{ml}$ rhBMP-4 before implantation.

\section{Implantation of prostheses}

After incubation with growth factors, the footplate anchor was placed on the stapes footplate using a transmeatal approach. After identifying the incus with the incudostapedial joint, the footplate anchor was inserted into the stapedial foramen which is formed by the crura. Before the footplate anchor was placed, a preparation needle was used to partly remove the middle ear mucosa by scratching on the bony footplate surface. The ossicular chain and/or the stapes suprastructure were removed if a correct placement of the implant was not secured. To prevent dislocation, the footplate anchor was covered with connective tissue. All procedures were performed under general anesthesia using $50 \mathrm{mg} / \mathrm{kg}$ body weight ketaminhydrochlorid s.c. (Parker-Davis, Berlin, Germany) and $15 \mathrm{mg} / \mathrm{kg}$ body weight of $2 \%$ xylazinhydrochlorid s.c. (Bayer, Leverkusen, Germany).

\section{Experimental protocol}

A total of 23 animals (equals 46 ears) divided into five groups of eight to ten ears were studied following the same experimental protocol. In each animal, both ears were operated and studied separately.

\section{Assessments}

Timing of analyses. Measures of body weight and ABR were obtained in all groups on days-1, 1, 7, 14, 28, 56, and 84 p.i. Injections of fluorochromes for labeling of bone formation were performed on days 1, 7, 14, and 28 p.i. (Fig. 2). All further analyses were performed after killing at day 84 .

Fluorochrome sequential labeling. Polychrome labeling with four calcium-binding fluorescent dyes was used to assess new bone formation and remodeling after implantation (Rahn and Perren 1971). Tetracycline $(20 \mathrm{mg} / \mathrm{kg})$, alizarin complexon $(30 \mathrm{mg} / \mathrm{kg})$, calcein green $(20 \mathrm{mg} / \mathrm{kg})$, and xylenol orange $(90 \mathrm{mg} / \mathrm{kg}$; footplate anchor, Sigma-Aldrich, Munich, Germany) were administered in the mentioned postoperative intervals (Fig. 2) to allow evaluation of four sequential periods of bone growth. In brief, dyes were adjusted to $\mathrm{pH}$, sterilized, and administered subcutaneously in 5-ml depots bilaterally at the spine to minimize local toxic or inflammatory skin reactions.

To avoid cardiovascular side effects of an additional general anesthesia for intravenous polychrome application, we choose the subcutaneous application as described by another group (Pautke et al. 2005).

Auditory brainstem responses. BERA was used to detect the ABR performed under ketamine/xylazine 


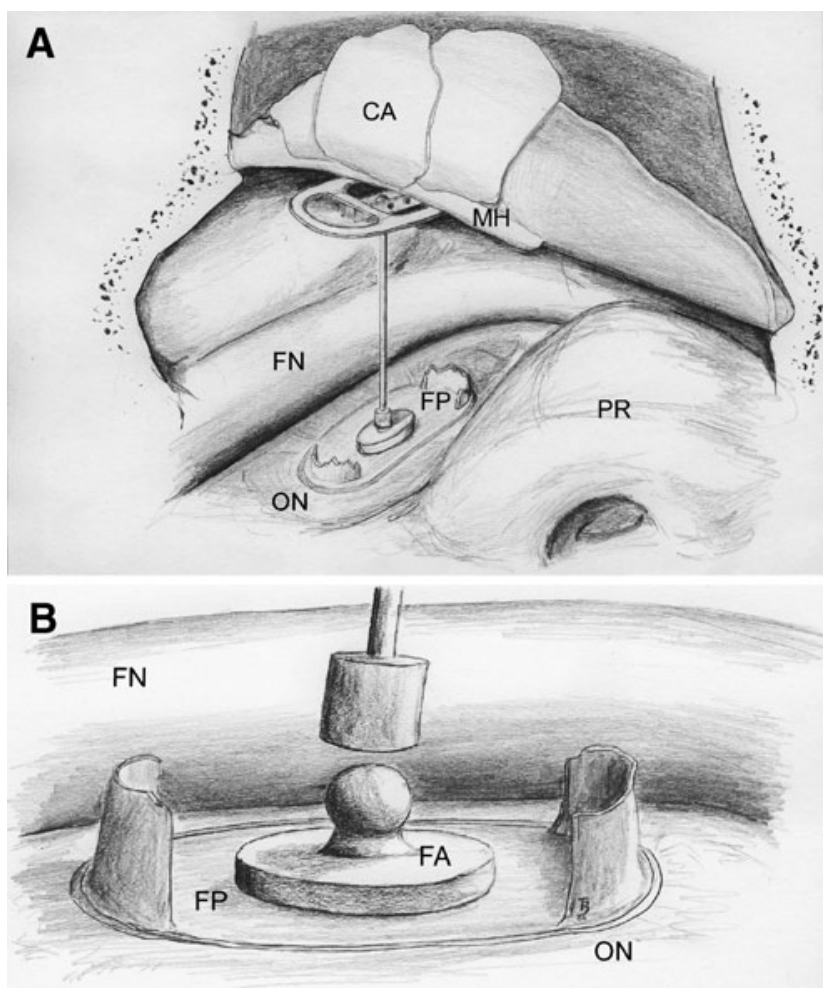

FIG. 1. The second surgical step of the compound prosthesis concept. The intraoperative situation shows the stapes footplate (FP) with the titanium footplate anchor (footplate anchor) in the oval niche $(O N)$. The proper prosthesis is coupled to the footplate anchor and placed under the malleus' handle and the reconstructed tympanic membrane $(C A)$. Mounting of the proper prosthesis is planned after the osseointegration of the surface coated footplate anchor which was placed in the first surgical step (not shown). $C A$ cartilage slices, $F N$ facial nerve, $P R$ promontory.

sedation and timed according to the experimental protocol as mentioned above (Fig. 2). Testing was performed using monaural stimuli presented at a rate of ten times per second, and a total of 1,024 trials were averaged at each intensity level. A computerbased signal averaging system was used to collect ABR data. Sound was presented though an Etymotic ER-2 earphone which was placed directly in the investigated ear canal and the contralateral ear for masking. Bone conduction was excited using a $\mathrm{KH} 70 / 5$ vibrator placed on the sheep's forehead. The tympanic membrane was examined with an otoscope prior to earphone placement. Needle electrodes were used to record brain potentials differentially. The active lead was positioned at the vertex and referred to the second electrode at the tip of the mastoid. Stimulus intensities were expressed in decibels sound pressure level (SPL), peak equivalent. Animals were presented with a stimulus (clicks) intensity series that was initiated at $70 \mathrm{~dB}$ SPL for air conduction and $50 \mathrm{~dB}$ for bone conduction. Stimulus intensity was progressively decreased in $10 \mathrm{~dB}$ increments and reached a minimum of $10 \mathrm{~dB}$ SPL. Threshold was defined as the lowest intensity at which a reproducible response was seen that contained at least two peaks and had amplitudes of at least $0.5 \mu \mathrm{V}$. Pretreatment ABR thresholds were used as baselines for comparison of post-treatment ABR.

\section{Histological examinations}

After killing, both temporal bones along with the medial scull base were removed and fixed in $4 \%$ neutral buffered formalin until further preparation. To target the implant, the tympanic cavity was opened and the stapes footplate along with the footplate anchor was marked by placing a destination cylinder on the oval niche. After conventional embedding in methylmetacrylate (Technovit 7200, Kulzer, Wehrheim, Germany), the excrescent material was ground until a cylindrical shape ( $7 \mathrm{~mm}$ in diameter) of the probe was achieved. Thirty specimens, six per experimental group, were applied for micro-CT scans before being used for histological preparation.

Due to the small implant size, a modified grounding technique according to Donath was used (Donath and Breuner 1982). Undecalcified specimens were then grounded sagittally along the $x$-axis of the footplate on a roll grinder containing sandpaper (Exakt-Apparatebau, Norderstedt, Germany) until the footplate and footplate anchor appeared at the specimens' grounding surface. The specimens were

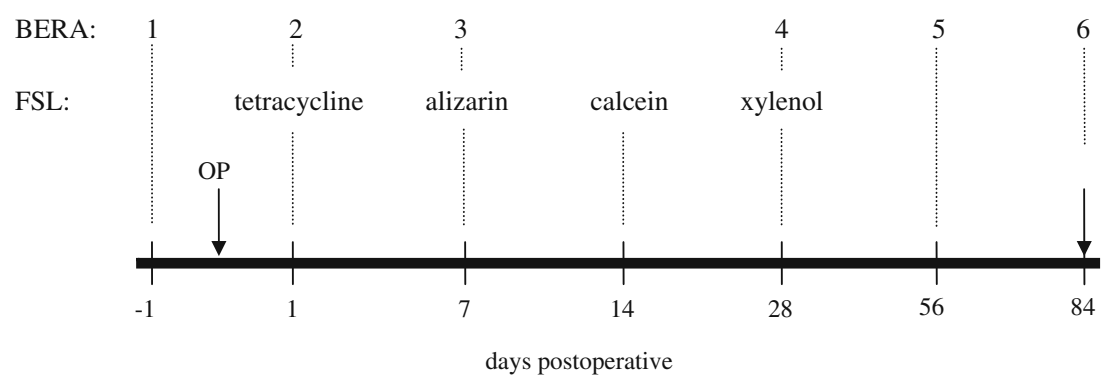

FIG. 2. Experimental protocol of the study. Animals' hearing was assessed by objective audiometry (BERA) during the study. After the footplate anchor implantation on day $0(O P)$, fluorescence sequence labeling $(F S L)$ was performed by subcutaneous administration of fluorescent dyes. All animals were killed on day 84 . 
then analyzed using a fluorescence microscope (BX 61, Olympus, Hamburg, Germany) with a BH2 RFL T3 halogen lamp (Olympus). An appropriate fluorescence filter (U-MNB2, Olympus) was used to excite fluorochromes. Fluorescence microscopic images were acquired using a conventional digital camera with a fluorescence mode as control (Color view II, Olympus).

As a next step, the histological sections were stained according to Masson-Goldner, and the reflected light microscopic image was taken again using the digital camera set at the identical localization (BX 61, Olympus) connected to a computerized digital image scanning system of histomorphometry (Cell F, Olympus). Thereafter, the specimen was sequentially processed starting with a new reduction of $100 \mu \mathrm{m}$ by grounding and reentering the aforementioned workup. By this technique, three to four images per specimen showing the footplate anchor bone interface were obtained.

For each section, the bone implant contact and the tissue quality (connective tissue/bone) were assessed. In case of a direct bone implant contact with new bone formation, the implant surface length that was in contact with the footplate surface was measured.

\section{Synchrotron radiation-based computed microtomography}

Synchrotron radiation enables quantitative microtomographic analyses that do not suffer from beamhardening artifacts. Synchrotron radiation-based computed microtomography $(\mathrm{SR} \mu \mathrm{CT})$ scans were obtained from 30 specimens, six per experimental group. The measurements were performed at HASYLAB BW2 (DESY, Hamburg, Germany). With a photon energy of $28 \mathrm{keV}$ and an image size of $512 \times$
512 pixels, 295 projections per implant were recorded. A filtered backprojection algorithm was used to obtain the three-dimensional data of X-ray absorption for the samples. The visualization of the reconstructed data was done with AMIRA (Mercury Computer Systems, Chelmsford, MA, USA).

The implant positions with respect to the stapes footplate and the bone implant contact areas were quantitatively determined.

\section{Statistical analyses}

Statistical analyses were performed using the SAS software package. Descriptive data are expressed as mean $\pm \mathrm{SD}$. Between-group comparisons were performed using the Student's $t$ test after testing for normal distribution of values. A $p$ value of $<0.05$ was considered significant.

\section{RESULTS}

\section{Effects of coated implants on inner ear function}

The ossicular chain and/or the stapedial suprastructure had to be removed in 13 of $46(28 \%)$ operated ears. Therefore, a postoperative follow-up of air conduction thresholds was not possible in all individuals and was consequently not used to assess the animal's hearing. Nevertheless, the mean air conduction threshold in all animals on day -1 was $33.0 \pm 9.4$ and $23.8 \pm 17.4 \mathrm{~dB}$ for bone conduction, respectively. Postoperatively, the mean bone conduction threshold in all experimental groups showed no statistical significant difference compared with the baseline measurements on day -1 (Table 1 ).

\section{TABLE 1}

\begin{tabular}{|c|c|c|c|c|c|c|}
\hline \multicolumn{7}{|c|}{ Bone conduction thresholds in experimental groups (means and changes from baseline) } \\
\hline & \multicolumn{6}{|c|}{ Postoperative days } \\
\hline & -1 & 1 & 7 & 28 & 56 & 84 \\
\hline Group I (Ti) & $39.4 \pm 14.3$ & $\begin{array}{l}31.3 \pm 8.4 \\
-8.1 \pm 12.5\end{array}$ & $\begin{array}{r}28.1 \pm 13.6 \\
-11.3 \pm 13.3\end{array}$ & $\begin{array}{l}38.8 \pm 13.6 \\
-0.6 \pm 17.8\end{array}$ & $\begin{array}{r}41.3 \pm 14.6 \\
1.9 \pm 16.9\end{array}$ & $\begin{array}{l}33.8 \pm 7.4 \\
-5.6 \pm 10.5\end{array}$ \\
\hline Group II (Ti+Coll I+CS) & $29.0 \pm 3.2$ & $\begin{array}{c}29.0 \pm 12 \\
0.0 \pm 11.6\end{array}$ & $\begin{array}{l}26.5 \pm 8.2 \\
-2.5 \pm 7.9\end{array}$ & $\begin{array}{r}33.0 \pm 12.5 \\
4.0 \pm 12.7\end{array}$ & $\begin{array}{r}26.0 \pm 8.4 \\
-3 \pm 9.5\end{array}$ & $\begin{array}{r}31.0 \pm 12 \\
2 \pm 11.4\end{array}$ \\
\hline Group III (Ti+Coll I+CS+BMP4) & $43.3 \pm 10.3$ & $\begin{array}{r}31.7 \pm 14.7 \\
-11.7 \pm 20.4\end{array}$ & $\begin{array}{r}31.7 \pm 14.7 \\
-11.7 \pm 20.4\end{array}$ & $\begin{array}{r}33.3 \pm 10.3 \\
-10 \pm 16.7\end{array}$ & $\begin{array}{r}33.3 \pm 22.5 \\
-10 \pm 30.3\end{array}$ & $\begin{array}{l}41.7 \pm 16.0 \\
-1.7 \pm 17.2\end{array}$ \\
\hline Group IV (Ti+Coll II+DC) & $28.3 \pm 7.5$ & $\begin{array}{c}21.7 \pm 4.1 \\
-6.7 \pm 8.2\end{array}$ & $\begin{array}{r}23.3 \pm 5.2 \\
-5 \pm 8.4\end{array}$ & $\begin{array}{r}33.3 \pm 5.2 \\
5 \pm 8.4\end{array}$ & $\begin{array}{r}28.3 \pm 4.1 \\
0 \pm 6.3\end{array}$ & $\begin{array}{l}26.7 \pm 5.2 \\
-1.7 \pm 7.5\end{array}$ \\
\hline Group V (Ti+Coll II+DC+TGF $\beta 1)$ & $30.0 \pm 5.0$ & $\begin{array}{l}22.2 \pm 4.4 \\
-7.8 \pm 4.4\end{array}$ & $\begin{array}{l}22.2 \pm 4.4 \\
-7.8 \pm 6.7\end{array}$ & $\begin{array}{r}31.1 \pm 6.0 \\
1.1 \pm 3.3\end{array}$ & $\begin{array}{r}31.3 \pm 8.4 \\
1.3 \pm 9.9\end{array}$ & $\begin{array}{r}30 \pm 8.7 \\
0.0 \pm 8.7\end{array}$ \\
\hline
\end{tabular}

There is no significant decrease in bone conduction thresholds in all groups, indicating that neither the operation nor the implant coating had negative effects on the animals hearing. Data shown are mean \pm SEM, absolute thresholds (first line), and change from baseline (second line), respectively. 


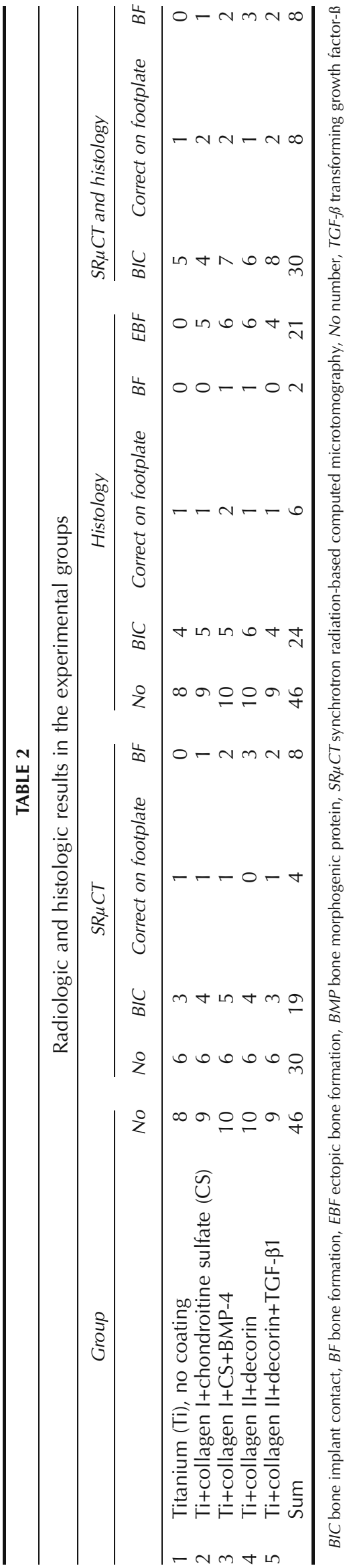

\section{Imaging techniques}

Table 2 gives a detailed overview on the imaging results stratified for the experimental groups. Since not all specimens were investigated by both SR $\mu \mathrm{CT}$ and histology, the number of correctly placed or dislocated implants and of those with a bone implant contact or bone formation vary in the specific method results. Thirty specimens were assessed with SR $\mu \mathrm{CT}$, whereas all specimens underwent histological workup. Therefore, there are implants with histologically observed findings that were not assessed by SR $\mu \mathrm{CT}$. On the other hand, there are specimens with radiographically detected findings that could not be verified on the histological sections because of the low number of realizable sections and the resulting position of the cutting planes. Therefore, the total numbers are not a summation of the radiographical and histological results but the number of implants showing specific findings in either $\mathrm{SR} \mu \mathrm{CT}$ and/or the histological sections.

\section{Loss rate and dislocation of implants}

Four footplate anchors were lost during surgery in a flow of secretions in the tympanic cavity or uncontrolled suction. Two could be replaced, whereas two ears remained without any implant or further stapes manipulation. Implants were considered as dislocated if they were not positioned on the stapes, facing the footplate with their undersurface. In contrast to the footplate anchor that had no detectable contact to the stapes at all, there was a subgroup of implants which had contact to the footplate with parts other than the coated undersurface or were in contact at other sites of the stapes (e.g., crura). Although these footplate anchors were in contact to the stapes, they had to be considered as functionally dislocated.

In the SR $\mu \mathrm{CT}, 19$ of 30 specimens showed a direct bone implant contact to any part of the stapes. The analyses of the histological sections showed that a direct bone implant contact to the stapes was detected in 24 specimens. Summing up the numbers of implants that had a detectable bone implant contact to the stapes in at least one imaging technique, 30 footplate anchors had bony contact to the stapes. In conclusion, the total number of correct positioned implants facing the footplate is six; 24 implants were functionally dislocated and 16 had no contact to the stapes at all.

\section{Induction of new tissue formation}

In all histologically assessed specimens, the footplate anchor was surrounded by newly formed tissue. No 
inflammatory response or adverse reactions were seen around the implants.

Two of six correct positioned footplate anchors were osseointegrated by newly formed bone. In these cases, a directed osteoneogenesis from the footplate toward the implant and its integration in the newly formed osteoid could be observed. The newly formed tissue could be clearly identified as bone in both the conventional histology with Masson-Goldner stain (Figs. 3A and 4A) and the fluorochrome sequential labeling (FSL; Figs. 3B and 4B). In the latter, an interposition of fibrous tissue in the contact area could be excluded. The integrated footplate anchors were coated with coll-I/CS/BMP (group 3) and collII/DC (group 4), respectively. The amount of newly formed bone was measured in the histological section showing the maximum extend and accounted for $4.6 \times 10^{-5} \mathrm{~mm}^{2}$ (coll-I/CS/BMP) and $3.2 \times 10^{-5} \mathrm{~mm}^{2}$ (coll-II/DC).

Furthermore, ectopic bone formations on the footplate anchor's surface (coated and uncoated areas) were observed in $21(46 \%)$ cases. These thin layers of newly formed osteoid had no direct contact to the stapes in the histological sections. Due to the footplate anchor size and the degrading histological specimen workup, a direct stapes contact of the ectopic bone formations in other cutting planes could not be proven. Ectopic bone formations were seen on 12 footplate anchors (26\%) that had any sort of detectable contact to the stapes and on nine footplate anchors $(20 \%)$ that were completely dislocated from the stapes. The implant surface coatings in the ectopic bone formations were distributed over experimental groups 2 to 5 without any predominance $(n=4$ to 6 ). On the non-coated titanium implants (group 1), no ectopic bone formations were seen. Consequently, in 23 out of 38 coated footplate anchors, either a contact bone formation (two cases) or ectopic bone formations (21 cases) were detected (Table 2).

\section{Comparison of radiographic and histological methods}

Direct comparison of regions with newly formed bone in $\mathrm{SR} \mu \mathrm{CT}$ scans and histological sections was possible in two specimens. In these cases, the radiographically and histologically determined site and extend show corresponding results of newly formed bone. The advantage of the radiographic technique allowed the evaluation of the whole contact area. The coll-I/DC/ BMP-4 coated implant was embedded at its edge with a bone isodense brace-shaped formation on approx-
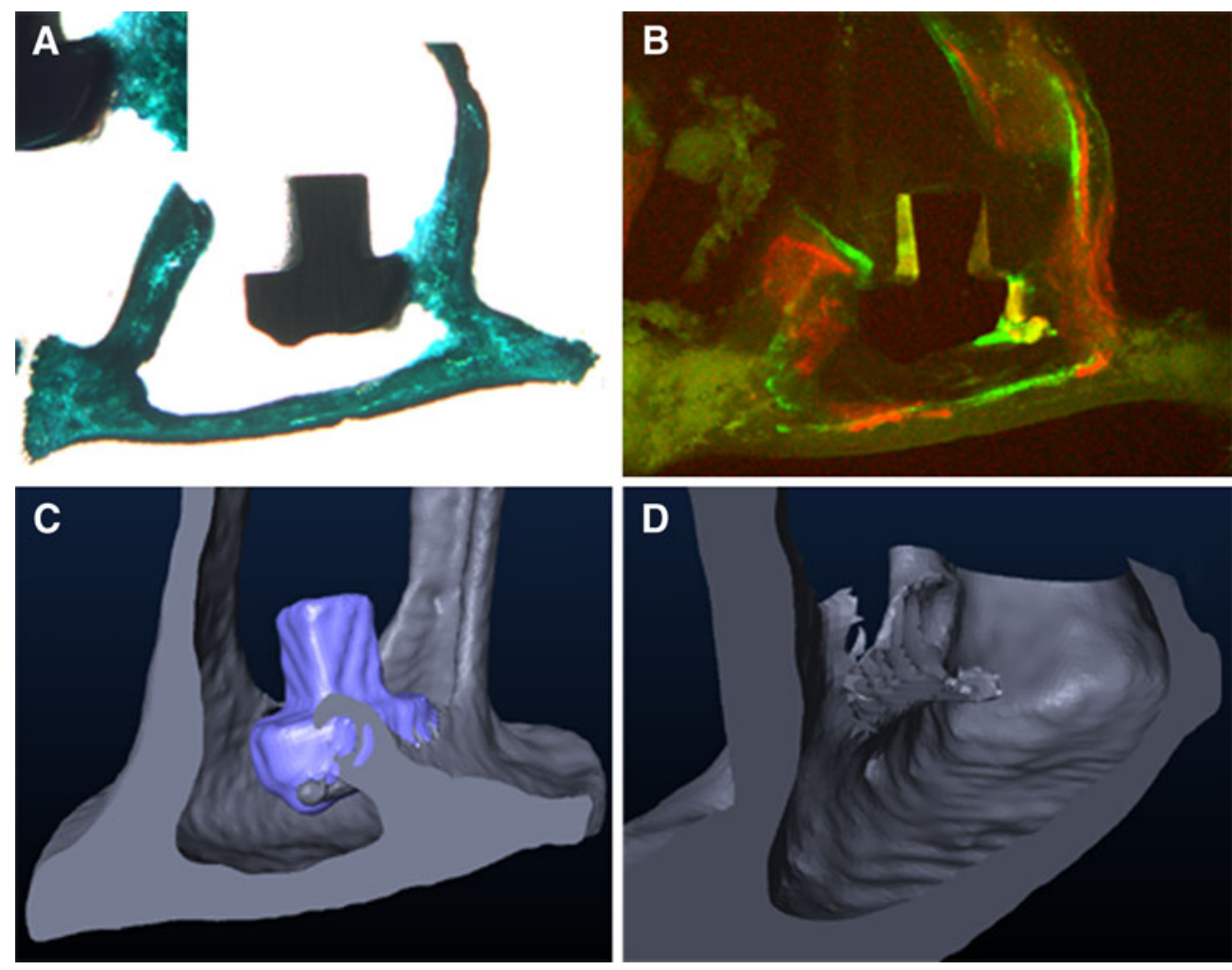

FIG. 3. Osseointegration of a collagen I+decorin+bone morphogenic protein 4-coated titanium footplate anchor. A The histological section shows the implant embedded at its edge with a bone isodense brace-shaped formation. B The fluorochrome sequence labeling clarifies the new bone a formed between days 21 and 28

after implantation. The three-dimensional reconstruction of the specimen (C) illustrates the brace-like shape of the bone formation. D Again, the computed implant elimination brings out the greater extend of osseointegration along the implants edge. A view from the rear is illustrated for better visualization of the newly formed bone. 

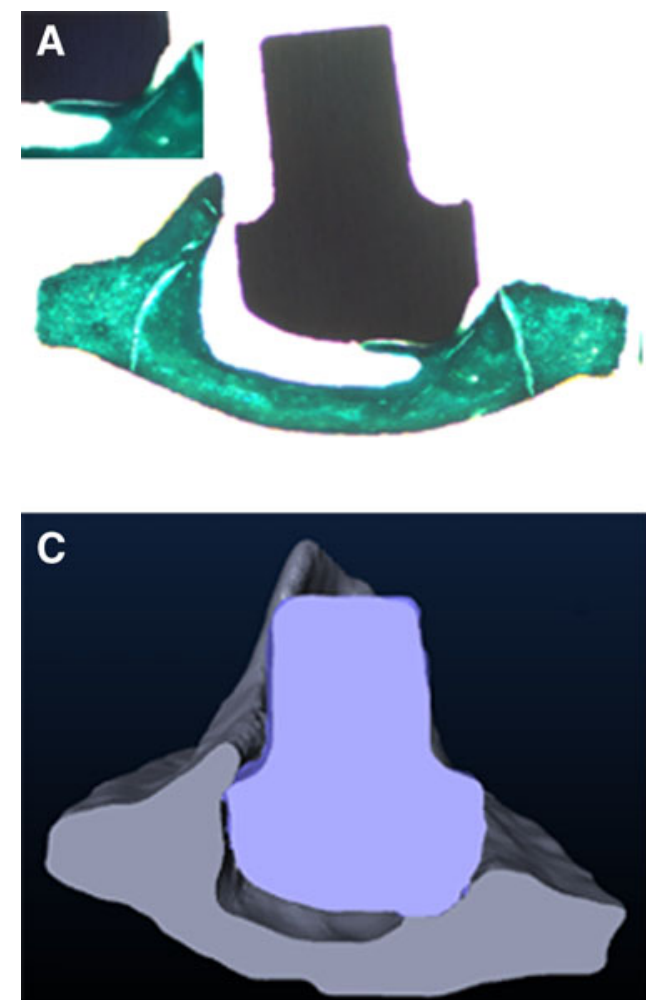

FIG. 4. Osseointegration of a collagen II+chondroitin sulfatecoated titanium footplate anchor. A The histological section shows the full embedding of a single contact bump with bracket-shaped osteoid. B The fluorochrome sequence labeling clarifies the new bone as formed between days 21 and 28 after implantation. Note also the accumulation of fluorochromes in the footplate as the result

imately $1 / 4$ of the footplate anchor circumference (Fig. 3C, D). Analogously, the coll-II/CS-coated implant showed a full embedding of one contact bump at the footplate anchor undersurface, illustrated by a slim bone isodense bracket along the implant and an additional bone limb at the posterior implant end (Fig. 4C, D). Identical bone isodense formations between the footplate anchor and the stapes were identified in another eight specimens where a direct bone implant contact was radiographically documented.

\section{DISCUSSION}

In otologic surgery, implant stability is of paramount importance to ensure good postoperative sound conduction. Although many factors influence the hearing outcome, the stable contact between the prosthesis and the ossicular remnants is crucial. Patch or clamp techniques cannot be applied on the stapes footplate, and thus, the prosthesis foot fixation must be achieved by surrounding of connective tissue or a cartilage guide (Hüttenbrink et al. 2004). Recently, a footplate implant for TORP, so-called $\Omega$ connector,
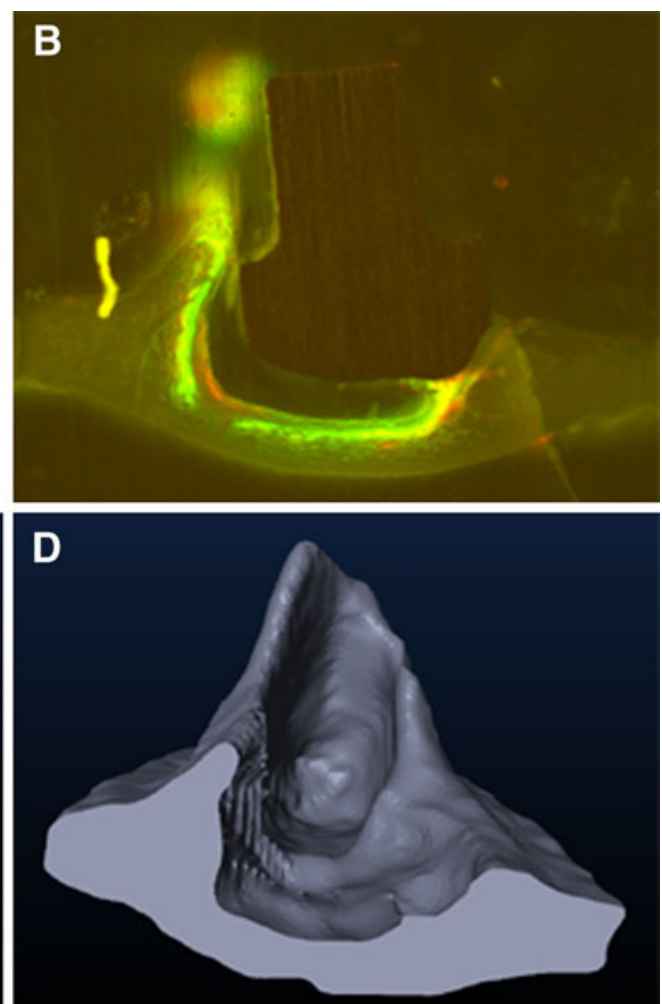

of bone turnover. The three-dimensional reconstruction of the specimen $(\mathbf{C})$ bares the greater extend of osseointegration as assumed by the Two-dimensional histological section. After computed elimination of the implant, the negative pattern of the footplate anchor indicates the areas of newly formed bone particularly toward the posterior stapes crurum (D).

was described (Schmid et al. 2009). This device also avoids a horizontal dislocation of the prosthesis on the footplate, but the interposition of newly formed connective tissue and the risk of a prosthesis uplift in perpendicular direction are still not eliminated.

Possible reasons for the inability to bony integrate titanium and other prosthesis materials on the stapes footplate are its thickness and the constant relative motions in the prosthesis footplate contact zone. The thickness of the human footplate ranges from 100 to $150 \mu \mathrm{m}$, and a closer look at its microscopic structure reveals that merely $1 / 3$ consists of bone, whereas the rest is formed by a cartilage layer (Oesterle 1933). The bony layer, facing the middle ear cavity, is configured by extracellular matrix, mainly collagen fibers, containing only a few osteocytes. Therefore, placing a prosthesis on the footplate center is presumably not the adequate stimulus to start a bony integration upon a $150-\mu \mathrm{m}$-thick bone layer with insular bone cells. Additionally, otosurgeons report contrary findings after footplate fractures since they describe both persisting fractures even years after the initial intraoperative diagnosis and a complete restoration of the bony footplate surface. The report of an accidentally extracted stapes in revision tympano- 
plasty caused by a bony integrated titanium TORP on the footplate (Sudhoff et al. 2005) supports the assumption of bone remodeling on the stapes footplate, and animal studies have shown a calcium and phosphorous uptake in the bone labyrinth in rats and dogs (Steller 1954). Altogether, the microscopic stapes structure and the clinical findings account for the general potential of footplate osteoblasts to form new bone even though the conditions for new bone formation and osseointegration are unfavorable.

To overcome this disadvantage, we set about a compound prosthesis concept in two steps: the footplate anchor is placed on the footplate in the first operation and the columella prosthesis in a second step after the footplate anchor is bony fixed by newly formed tissue. Thus, the staged procedure provides the necessary time interval the implanted footplate anchor needs to maintain on the footplate without any relative movements. Furthermore, the applied implant coating induces the formation of new bone in the footplate anchor footplate contact zone.

Upon histological examination, two of six footplate anchors with direct bone implant contact in the correct position were fixed on the footplate by newly formed bone. Masson-Goldner staining and FSL verified the tissue as bone and additionally as principally formed between days 21 and 28 according to the time points of fluorochrome administration. To our knowledge, this is the first reported successful attempt to induce and realize a controlled osseointegration of titanium prostheses on the stapes footplate. These implants were coated with coll-I/CS/BMP-4 and coll-II/DC, respectively. Histologically, an osseointegration was only detected in these two implanted footplate anchors, but the morphological characteristics of the bone implant contact area in these two specimens were identical compared to another six implants where an osseointegration is most likely from the $\mathrm{SR} \mu \mathrm{CT}$ data.

The analyses of the obtained SR $\mu \mathrm{CT}$ scans showed that the percentage of radiographically covered footplate anchor's surface by bony structures (bone implant contact) varies between $9 \%$ and $52 \%$ for the coated implant surfaces. Focusing on the two specimens that were histologically proven to be integrated in newly formed bone, the radiographically detected bone implant contact area accounts for $32 \%$ $\left(0.134 \mathrm{~mm}^{2}\right)$ in the coll-II-coated footplate anchor and for $44 \%\left(0.187 \mathrm{~mm}^{2}\right)$ in the coll-I/BMP-4-coated footplate anchor (related to the whole coated implant surface). We have shown previously that a minimal bony fixation of one bump up to $60 \%$ of its height was calculated to be necessary to endure all arising forces in the accretion zone (Neudert et al. 2007). Since a single bump accounts for $5 \%\left(0.02 \mathrm{~mm}^{2}\right)$ of the implant surface, the here achieved osseointegrated area exceeds the required percentage of osseointegration by $578 \%$ and 807 , respectively.

Due to the small implant and footplate sizes, the low number of histologically detected osseointegrated implants is most likely caused by experimental limitations, assuming that an additional six implants must radiographically be highly considered to be fixed to the footplate by newly formed bone.

Ectopic bone formations were detected in another 21 specimens either as a consequence of new bone formation upon stem cell differentiation in the postoperative hemorrhagic middle ear effusion or by direct bone growth along the implants.

There was no difference in integration patterns of the administered fluorescent dyes depending on the implant coatings. In the two specimens with histologically verified osseointegration, the bone implant contact area features calcein and xylenol and marks the bone to be formed within days 14 and 28 after implantation. This must be considered when planning the second operation for implanting the proper columella prosthesis onto the integrated footplate anchor. Our results suggest that the footplate anchor should not be loaded within 4 weeks after implantation to ensure a sufficient bony integration. Given that the FSL showed an integration of at least two of four fluorescent dyes in all stapes footplates, this clearly indicates a successful formation of new bone in this region within the observed interval. It was also shown that this bone formation was no effect of the stapes manipulation itself. In two ears, the stapes suprastructure was not removed and an implant could not be placed as the footplate anchor was lost in the middle ear cavity. The FSL documented a bone formation between days 14 and 28 in these cases too. Thus, these results clearly indicate a bone turnover and new bone formation within the stapes footplate in this animal model. Whether these findings can be transferred to human conditions remains open. However, the Merino sheep is a mammal and widely used and established in experimental setups to investigate bone turnover in metabolic bone disease (Sigrist et al. 2007) and located fracture healing or integration of prostheses in the skeleton other than the middle ear (Cancedda et al. 2007). Adverse mechanisms like the potential of regeneration of ossicular remnants, as known in rodents (Dost et al. 2002), were neither reported in literature nor seen in our study. Therefore, we assume our data to be comparable to adult human conditions in metabolic concerns.

The low rate of footplate anchors that remained in the correct position on the footplate until study termination delimits the explanatory power of our results. Conclusions on the effect of the different implant coatings regarding their influence on the process of osseointegration cannot be drawn. Since 
the two bony integrated implants were coated by collI/CS/BMP-4 and coll-II/DC, it appears as if the application of a collagen matrix is able to support an osteoneogenesis on the stapes footplate. BMP induces osteogenic cell differentiation in vitro as well as bone defect healing in vivo. Enhancing the binding of endogenic BMP by collagen matrices with CS creates an osteogenic microenvironment that allows multipotent cell progenitors to migrate to the area of injury and to proliferate and differentiate toward the osteogenic lineage (Chen et al. 2004). The BMP activity is related to the cytokine's ability to mobilize the patient's own stem/progenitor cells to result in the healing process; this ability was demonstrated for human (Chaudhary et al. 2004) and for rat bone marrow stromal cells (Hu et al. 2005). In our study, the ectopic bone formation could be the result of activated osteogenic progenitor cells by either endogenic or the administered recombinant growth factors. Unfortunately, the high footplate anchor dislocation rate precludes any further statement regarding possible differences in the administered implant coatings.

Nevertheless, the data presented here clearly show the general ability of the stapes footplate to integrate titanium implants with bone when coated with artificial extracellular matrices from collagen and proteoglycans/glycosaminoglycans in a mammalian organism. In this regard, an excessive new bone formation with fixation of the stapes footplate in the oval niche was not observed. Unfortunately, the chosen footplate anchor design revealed disadvantages concerning its dislocation from the footplate after implantation. The footplate anchor design already underwent decisive modifications to ensure a stable positioning on the stapes footplate, and further experiments are in process to evaluate the influencing factors for osseointegration. New prostheses concepts combining different components of ossicular reconstruction methods are conceivable once a firm and stable fixed point on the footplate is established. Similar to using a kit of precisely defined components, ossicular reconstruction could be improved by individual assemblies built on top of an osseointegrated footplate anchor. The importance of the results becomes even clearer when future applications of a controlled osseointegration in the middle ear are considered. Stable prosthesis coupling to other sites of ossicular remnants could be improved by osteoinductive surface coatings as well as the implantation of active middle ear implants and their coupling to bony middle ear structures. Following this successful attempt of inducing a controlled and intended osseointegration on the footplate, new options in otologic surgery become available.

\section{ACKNOWLEDGMENTS}

The authors wish to thank Timothy Douglas for development of the implant coating system and implant coating. We would further like to thank Dr. Kathrin Speckl, Dr. Roland Jung, and Elke John for their broad assistance in all animal experiments. We are grateful to Dr. Ludwig Kant (NRU) and Thomas Burkhardt (IWU) for manufacturing and providing the titanium implants and Dr. Felix Beckmann for his help with the SR- $\mu$ CT measurements. We also greatly appreciate Toni Sanders linguistic support and Anton Poznyakovskiys help with the AMIRA figures.

This work was supported by a grant from the Bundesministerium für Bildung und Forschung (03I4034A), Beamtime at Hasylab supported by grant II-03-065.

The authors certify that they have not entered into any agreement that could interfere with their access to the data on the research nor upon their ability to analyze the data independently, to prepare manuscripts, and to publish them.

\section{REFERENCES}

Becker D, Geissler U, Hempel U, Bierbaum S, Scharnweber D, Worch H, Wenzel KW (2002) Proliferation and differentiation of rat calvarial osteoblasts on type I collagen-coated titanium alloy. J Biomed Mater Res 59:516-527

Bessho K, Carnes DL, Cavin R, Chen HY, Ong JL (1999) BMP stimulation of bone response adjacent to titanium implants in vivo. Clin Oral Implants Res 10:212-218

Branemark PI, Hansson BO, Adell R, Breine U, Lindstrom J, Hallen O, Ohman A (1977) Osseointegrated implants in the treatment of the edentulous jaw. Experience from a 10-year period. Scand J Plast Reconstr Surg Suppl 16:1-132

Cancedda R, Giannoni P, Mastrogiacomo M (2007) A tissue engineering approach to bone repair in large animal models and in clinical practice. Biomaterials 28:4240-4250

Chaudhary LR, Hofmeister AM, Hruska KA (2004) Differential growth factor control of bone formation through osteoprogenitor differentiation. Bone 34:402-411

Chen D, Zhao M, Mundy GR (2004) Bone morphogenic proteins. Growth Factors 22(4):233-241

Donath K, Breuner G (1982) A method for the study of undecalcified bones and teeth with attached soft tissues. The Sage-Schliff (sawing and grinding) technique. J Oral Pathol 11:318-326

Dost P, Ellermann S, Missfeldt NN, Leyden PJ, Jahnke K (2002) Reconstruction of the stapes superstructure with a combined glass-ceramic (Bioverit) implant in guinea pigs. ORL J Otorhinolaryngol Relat Spec 64:429-432

Douglas T, Heinemann S, Mietrach C, Hempel U, Bierbaum S, Scharnweber D, WORCH H (2007A) Interactions of collagen types I and II with chondroitin sulfates A-C and their effect on osteoblast adhesion. Biomacromolecules 8:1085-1092

Douglas T, Hempel U, Mietrach C, Heinemann S, Scharnweber D, WORCH H (2007в) Fibrils of different collagen types containing immobilised proteoglycans (PGs) as coatings: characterisation and influence on osteoblast behaviour. Biomol Eng 24:455-458

Eiber A, Freitag HG, Schimanski G, Zenner HP (2000) On the coupling of prostheses to the middle ear structure and its influence on sound transfer. In: Rosowski JJ, Merchant SN (eds) Second International Symposium on Middle-Ear Mechanics in 
Research and Otosurgery. Boston, MA, USA. Kugler, The Hague, The Netherlands

FisCH U, MAY J (1994) Tympanoplasty, mastoidectomy and stapes surgery. Georg Thieme, Stuttgart

Hu Z, Peel SA, Ho SK, Sandor GK, Clokie CM (2005) Role of bovine bone morphogenetic proteins in bone matrix protein and osteoblast-related gene expression during rat bone marrow stromal cell differentiation. J Craniofac Surg 16:1006-1014

HÜtTensRink K-B (1994) Surgical treatment of chronic otitis media. III: middle ear reconstruction. HNO 42:701-718

Hüttenbrink K-B, Zahnert T, Beutner D, Hofmann G (2004) The cartilage guide: a solution for anchoring a columella-prosthesis on footplate. Laryngorhinootologie 83:450-456

JAHNKE K (1987) Extrusion of middle ear implants. Clin Otolaryngol Allied Sci 12:227-232

Katzke D, Steinbach E, Schödermaier C (1982) Zur ursache der wiederentfernung allogener anstoßtransplantate. Arch Otorhinolaryngol 235:525-528

Müller B, Bernhardt R, Weitkamp T, Beckmann $\mathrm{F}$, Bräuer $\mathrm{R}$, Schurigt U, Schrott-Fischer A, Glueckert R, Ney M, Beleites T, Jolly C, Scharnweber D (2007) Morphology of bony tissues and implants uncovered by high-resolution tomographic imaging. Int J Mater Res 98:613-621

Neudert M, Berner M, Bornitz M, Beleites T, Ney M, Zahnert T (2007) Osseointegration of prostheses on the stapes footplate: evaluation of the biomechanical feasibility by using a finite element model. J Assoc Res Otolaryngol 8:411-421

Oesterle F (1933) Über den feinbau der gehörknöchelchen und seine entstehung. Eur Arch Otorhinolaryngol 135:311-327

Pautke C, Vogt S, Tischer T, Wexel G, Deppe H, Milz S, Schieker M, Kolk A (2005) Polychrome labeling of bone with seven different fluorochromes: enhancing fluorochrome discrimination by spectral image analysis. Bone 37:441-445
Plester D, Hildmann H, Steinbach E (1989) Atlas der Ohrchirurgie. Kohlhammer, The Netherlands

RAHn BA, PerRen SM (1971) Xylenol orange, a fluorochrome useful in polychrome sequential labeling of calcifying tissues. Stain Technol 46:125-129

Rosso F, Giordano A, Barbarisi M, Barbarisi A (2004) From cellECM interactions to tissue engineering. J Cell Physiol 199:174 180

Schmid G, Steinhardt U, Heckmann W (2009) The omega connectora module for jointed coupling of titanium total prostheses in the middle ear. Laryngorhinootologie. doi:10.1055/s-00291231047. http://www.ncbi.nlm.nih.gov/pubmed/19639531? itool=EntrezSystem2.PEntrez.Pubmed.Pubmed_ResultsPanel. Pubmed_RVDocSum\&ordinalpos $=10$

Sigrist IM, Gerhardt C, Alini M, Schneider E, Egermann M (2007) The long-term effects of ovariectomy on bone metabolism in sheep. J Bone Miner Metab 25:28-35

Sмүтн GD (1983) TORPs- how have they fared after five years? J Laryngol Otol 97:991-993

Steller J (1954) Metabolism in the labyrinthine bones: experimental studies on metabolism of radiophosphorus and radiocalcium. Arch Ohren Nasen Kehlkopfheilkd 164:320-327

Sudhoff H, Lindner N, Gronemeyer J, Dazert S, Hildmann H (2005) Study of osteointegration of a titanium prosthesis to the stapes: observations on an accidentally extracted stapes. Otol Neurotol 26:583-586

Wolf C, Rössler S, Scharnweber D, Worch H (1999) Adsorption of collagen type I on titanium oxide surfaces. Proceedings of the 15th European Conference on Biomaterials, Bordeaux, p 55

Wong M, Eulenberger J, Schenk R, Hunziker E (1995) Effect of surface topology on the osseointegration of implant materials in trabecular bone. J Biomed Mater Res 29:1567-1575 